\title{
Dehydroepiandrosterone secretion in dairy cattle is episodic and unaffected by ACTH stimulation
}

\author{
Lieta Marinelli, Erminio Trevisi ${ }^{1}$, Laura Da Dalt, Mariacristina Merlo, Giuseppe Bertoni ${ }^{1}$ \\ and Gianfranco Gabai \\ Dipartimento di Scienze Sperimentali Veterinarie, Università di Padova, viale dell'Università, 16, Agripolis 35020 Legnaro (PD), Italy \\ ${ }^{1}$ Istituto di Zootecnica, Università Cattolica del Sacro Cuore, via Emilia Parmense, 84, 29100 Piacenza, Italy \\ (Correspondence should be addressed to G Gabai; Email: gianfranco.gabai@unipd.it)
}

\begin{abstract}
This paper describes the episodic release and response to adrenal stimulation of cortisol and dehydroepiandrosterone (DHEA) in cows. Observations made in samples taken every $10 \mathrm{~min}$ for $8 \mathrm{~h}$ (experiment 1 ) showed that plasma DHEA was significantly greater $(P<0 \cdot 001)$ than DHEA-S, and release of these steroids was episodic and variable between animals $(P<0 \cdot 01)$. No relationship was found between DHEA and cortisol. Significant $(P<0 \cdot 001)$ DHEA-sulphate (DHEA-S) versus cortisol $(R=-0 \cdot 264)$ and DHEA-S versus DHEA $(R=0 \cdot 200)$ correlations were found. DHEA and DHEA-S were not affected by a single ACTH challenge (experiment 2 ). In experiment 3 , cortisol and DHEA secretions in response to prolonged ACTH administration (every $12 \mathrm{~h}$ for 6 days) were studied. On day 7, the episodic cortisol and DHEA release and response to the opioid antagonist naloxone were studied in blood samples taken every $10 \mathrm{~min}$ for $8 \mathrm{~h}$. Animals were
\end{abstract}

injected with naloxone after $4 \mathrm{~h}$. A significant increase $(P<0.05)$ in mean circulating DHEA and DHEA pulse amplitude was observed during frequent sampling following ACTH treatment. DHEA and DHEA-S plasma concentrations were not affected following luteal regression (experiment 4). The effect of milk secretion around parturition on DHEA secretion was studied in dry and continuously milked cows (experiment 5). Plasma DHEA was significantly lower $(P<0 \cdot 05)$ in milked cows. In the cow, ACTH is not an important DHEA secretagogue. Adrenal contribution to plasma DHEA is scarce. Likely, the placenta is the most important source of DHEA, and the lactating mammary gland can affect circulating DHEA levels. Investigation about the DHEA biological role in cows should be focused around parturition.

Journal of Endocrinology (2007) 194, 627-635

\section{Introduction}

Dehydroepiandrosterone and its sulphate (DHEA-S) are secreted mainly by the adrenals of primates and serve as intermediates in the synthesis of androgens and estrogens in peripheral tissues (Labrie et al. 2005). They are the most abundant steroids in the human circulation, and they have been associated with several biological actions beneficial for human health and to the physiological and pathological phenomena connected to the ageing process (Roberts 1999, Labrie et al. 2005). In primates and rodents, DHEA is also a neurosteroid and it is produced within the CNS and in peripheral nerves, where it acts as an allosteric modulator of $\gamma$-amino butyric acid type A (GABA-A) and sigma receptors, and it is associated with several behavioural circumstances and neurodegenerative diseases (Baulieu 1998, Zinder \& Dar 1999, Maurice et al. 2001, Labrie 2005).

DHEA is implicated in the stress response (Zinder \& Dar 1999) and the adrenal of the adult human responds to stress with increased cortisol and diminished DHEA/DHEA-S secretion (Parker 1999). In rats, DHEA administration antagonises the effects of repeated immobilisation stress (Hu et al. 2000).
DHEA and its metabolites play a role in the immune system activation, counteracting the immunosuppressive action of cortisol (Loria et al. 1996). DHEA and DHEA-S have antiinflammatory effects probably mediated by their antioxidant properties, which prevent the activation of the pleiotropic transcription factor nuclear factor- $\mathrm{KB}(\mathrm{NF}-\kappa \mathrm{B}$; Iwasaki et al. 2004, Aragno et al. 2006). The antioxidant properties of DHEA have been demonstrated both in vivo (Pelissier et al. 2004) and in vitro (Bastianetto et al. 1999).

DHEA is also involved in lipid metabolism, showing protective effects against visceral fat accumulation and muscle insulin resistance development (Hansen et al. 1997) and, likely, it exerts its fat-reducing effects by increasing peroxisome proliferator-activated receptor- $\alpha(P P A R \alpha)$ gene expression and consequently inducing the expression of enzymes involved in fatty acid catabolism (Kochan \& Karbowska 2004).

Some findings raised our interest in the possible biological role of DHEA in the female bovine. First of all, the bovine mammary gland can convert DHEA into androstene- $3 \beta, 17 \beta-$ diol (AED; Belvedere et al. 1996). Then, plasma DHEA and AED concentrations are higher in late pregnancy and drop 
suddenly after parturition (Gabai et al. 2004). Finally, it was recently reported that cows with inflammatory foot lesions showed a $23 \%$ decrease in serum DHEA concentrations (Almeida et al. 2007), suggesting that DHEA is implicated in the inflammation process also in the bovine.

In an early study (Feher et al. 1977), it was observed that DHEA was present in the plasma of most mammals at nanomolar levels, and the concentrations of this steroid in animal plasma were similar or higher than those in human subjects. On the contrary, DHEA-S was present in human plasma at micromolar levels, and in plasma of rat, dog and pig at nanomolar levels, while it was undetectable in plasma of ruminants, guinea pig and rabbit.

In humans, DHEA and DHEA-S display a diurnal rhythm in parallel to that of cortisol (Lejeune-Lenain et al. 1987), and it was hypothesised that social activity and living habits may play a role in the synchronisation of diurnal DHEA/DHEA-S rhythms (Zhao et al. 2003). In the human, adrenal DHEA secretion is episodic and it is synchronous with cortisol release, while DHEA-S seems to be less synchronous with both DHEA and cortisol (Rosenfeld et al. 1975). In the human male, it was observed that the pulsatility of the corticotropic axis was transmitted to the secretory pattern of DHEA, and a partial synchronisation between testicular and adrenal pulsatile secretion was hypothesised (Lejeune-Lenain et al. 1987).

In humans, DHEA secretion is responsive to ACTH stimulation (Parker 1999, Arvat et al. 2000). However, in one study performed on healthy women (Azziz et al. 2001), it was observed that DHEA levels in response to ACTH stimulation were extremely variable among subjects. It is evident that the control of cortisol and DHEA secretion frequently diverges, and substances of pituitary origin other than ACTH were hypothesised to control adrenal androgen secretion (Odell \& Parker 1985).

To our knowledge, little information about DHEA and DHEA-S secretion in the dairy cow is available in the literature and, in particular, the adrenal contribution to DHEA secretion has been poorly investigated. The aim of this work was to extend the knowledge about the secretion of DHEA and DHEA-S in the dairy cow and, in particular, we aimed to study a) the pattern of DHEA and DHEA-S secretion, b) their response to exogenous ACTH administration and c) the effects of phase of the oestrus cycle (luteal versus follicular phases) and lactation on DHEA secretion.

\section{Materials and Methods}

\section{Animals and procedures}

Animals were trained to frequent handling for at least 1 month before each experiment, and animal health conditions were assessed by clinical examination during the whole experimental periods. All procedures were carried out in respect of the Italian legislation on animal care (DL n.116, 27/1/1992).

\section{Experiment 1: pattern of secretion}

This experiment was designed to study the episodic release of cortisol, DHEA and DHEA-S in the dairy cow. Six lactating non-pregnant Italian Friesian cows in their third month of lactation were used. Animals were housed in tie stalls, with almost constant climate conditions, daylight interval and feeddiet characteristics. Cows were individually fed ad libitum; forages (maize silage, alfalfa and grass hay) were offered in two meals (0730 and $1930 \mathrm{~h}$ ), and concentrate in eight meals by auto feeder. The cows were milked twice a day at 0500 and $1700 \mathrm{~h}$. Indwelling jugular catheters were inserted under local anaesthesia the day before the experiment, and blood samples were taken every $10 \mathrm{~min}$ for $8 \mathrm{~h}$, beginning at $1000 \mathrm{~h}$. Blood samples were kept refrigerated and plasma was obtained by centrifugation $\left(1500 \mathrm{~g}, 4^{\circ} \mathrm{C}, 15 \mathrm{~min}\right)$ within $30 \mathrm{~min}$.

\section{Experiment 2: response to single $A C T H$ challenge}

The experiment was carried out on four non-lactating nonpregnant multiparous Friesian cows to study the short-term response of cortisol, DHEA and DHEA-S to ACTH challenge. Animals were housed in individual boxes under natural light condition. Cows were fed once a day a total mixed ration (TMR) and had free access to water. The cows were randomly assigned to treatments according to a crossover experimental design, consisting of two experimental periods with a 40-day resting period in between. Treatments consisted of the i.m. administration of either saline (control; CTR) or an ACTH analogue (tetracosactrin acetate, Synacthen depot, Novartis Pharma; $0.5 \mathrm{mg}$; ACTH), and the treatment administration was referred as time 0 . The plasma concentrations of steroids were measured in plasma samples obtained from the jugular vein before $8 \mathrm{~h}$ and at 0,1 , 2, 4,9 and $12 \mathrm{~h}$ from treatment administration.

\section{Experiment 3: response to long-term ACTH administration}

The experiment was carried out on five non-lactating nonpregnant multiparous Friesian cows to study the response of cortisol and DHEA to long-term adrenal stimulation.

Animals were housed in tied stalls under natural light condition, were fed a standard diet consisting of hay and concentrate, and had free access to water. The experimental protocol consisted of a control phase (CTR) followed by a treatment phase (ACTH) with a resting period of at least 30 days in between. Animals received i.m. injections of either saline (CTR) or ACTH analogue (Tetracosactrin acetate, Synacthen depot, Novartis Pharma; $0.7 \mathrm{mg} /$ head/administration; ACTH) every $12 \mathrm{~h}$ per 6 days. The beginning of the treatment was indicated as day 0 . Blood samples were collected from the jugular vein in heparinised tubes and they were kept refrigerated until plasma was obtained by centrifugation $\left(1500 \mathrm{~g}, 4^{\circ} \mathrm{C}, 15 \mathrm{~min}\right)$ within $30 \mathrm{~min}$. Plasma was divided in aliquots and stored at $-20^{\circ} \mathrm{C}$ until required for analysis. Samples were taken with the same time intervals 
during the control and treatment phases to measure the concentrations of circulating cortisol and DHEA the day before and on days $0,1,4,5$ and 11 .

On day 7, the pulsatile cortisol and DHEA release and response to the opioid antagonist naloxone were studied in blood samples taken every $10 \mathrm{~min}$ for $8 \mathrm{~h}$. Indwelling jugular catheters were inserted under local anaesthesia the day before (day 6), and frequent sampling begun at $1000 \mathrm{~h}$. After $4 \mathrm{~h}$, the animals received an injection of naloxone hydrochloride $(1 \mathrm{mg} / \mathrm{kg}$ body weight i.v.; Sigma) dissolved in saline.

\section{Experiment 4: steroid variation around luteal regression}

This experiment was performed to study cortisol, DHEA and DHEA-S plasma concentrations around the period of the induced luteal regression, in order to study the ovarian influences on the plasma levels of these steroids. The experiment was carried out on four non-lactating nonpregnant multiparous Friesian cows housed in individual boxes under natural light condition. Cows were fed once a day a TMR and had free access to water. The cows were treated with two injections of prostaglandin (PG; $2 \mathrm{mg}$ cloprostenol, Estrumate, Schering-Plough, Uxbridge, UK) administered 11 days apart, and the second PG administration was referred as time 0. Plasma concentrations of cortisol, DHEA and DHEA-S were measured in samples taken from the jugular vein every $12 \mathrm{~h}$, from -60 to $0 \mathrm{~h}$, and at $4,12,24$, $36,48,60,110,120$ and $140 \mathrm{~h}$.

\section{Experiment 5: response to continuous lactation}

The last experiment was carried out in Holstein cows subjected to a 55-day dry period (CTRL, N=5) and continuously milked cows (MILK, N=6) to study the effect of mammary gland activity on the secretion of cortisol and DHEA. The animals were assigned to each experimental group to have homogeneous average milk yield, expressed as mature equivalent cow $(\mathrm{CTRL}=105 \pm 12$ ton; $\mathrm{MILK}=$ $109 \pm 14$ ton). The groups were placed in two distinct pens. The cows in the MILK group were milked until parturition. The cows of the CTRL group were dried-off 55 days before parturition. All cows had free access to water and were fed once daily. Blood samples were collected before parturition (at days $-60,-55,-40,-30$ and -7 ) and after parturition (at days 1, 7, 15, 30, 60 and 90).

\section{Hormone assays}

Plasma cortisol (C) was measured using a solid phase microtitre RIA after diethyl ether extraction (Gabai et al. 2006). All samples were assayed in duplicate. The intra- and inter-assay coefficients of variation $(\mathrm{CV})$ were 3.1 and $2.8 \%$ respectively.

Plasma DHEA was measured by the RIA described elsewhere (Gabai et al. 2004). The intra- and inter-assay CV were 8.7 and $10 \cdot 0 \%$ respectively.
Plasma DHEA-S concentrations were analysed by a solidphase microtitre direct RIA. Briefly, a 96-well microtitre plate Optiplate, Perkin-Elmer Life Science) was coated with antirabbit $\gamma$-globulin serum raised in a goat, incubating overnight the antiserum diluted $1: 1000$ in $0.15 \mathrm{mM}$ sodium acetate buffer, $\mathrm{pH} 9$, at $4{ }^{\circ} \mathrm{C}$. The plate was then washed twice with PBS, $0 \cdot 1 \%$ BSA, $\mathrm{pH} 7 \cdot 4$ (RIA buffer) and incubated overnight at $4{ }^{\circ} \mathrm{C}$ with $200 \mu \mathrm{l}$ of the anti-DHEA-S serum diluted 1:8000. The antiserum (Spi Bio, Montigny, France) was raised in the rabbit against DHEA-S-7-carboxymethyloxime-BSA and showed the following cross reactions: DHEA-S $100 \%, \Delta 4$-androstenedione $0 \cdot 2 \%$, DHEA, testosterone and androsterone $0 \cdot 01 \%$. The plate was carefully washed with RIA buffer, and standards (1.56$400 \mathrm{pg} /$ well $/ 50 \mu \mathrm{l})$, quality control, unknown samples $(20 \mu \mathrm{l})$ and tracer $\left(1,2,6,7^{3} \mathrm{H}-\mathrm{DHEA}-\mathrm{S}\right.$, Perkin-Elmer Life Sciences, $30 \mathrm{pg} /$ well $/ 10 \mu \mathrm{l}$, specific activity: $2927 \mathrm{GBq} / \mathrm{mmol}$ ) were added. The final volume of the reaction mixture was adjusted to $200 \mu \mathrm{l}$ with RIA buffer. The plate was incubated overnight at $4{ }^{\circ} \mathrm{C}$, the incubation mixture was decanted and wells washed with RIA buffer, added with $200 \mu$ scintillation cocktail (Microscint 20, Perkin-Elmer Life Sciences) and counted on the $\beta$-counter (Top-Count, Perkin-Elmer Life Sciences). The DHEA-S RIA was validated by performing parallelism and recovery tests. Parallelism data were expressed as the regression curve between observed hormone concentrations and the reciprocal of the dilution factors of serially diluted plasma samples $\left(\left(H_{\mathrm{obs}}\right)=a \times 1 / \mathrm{d} f+b\right)$. The assay showed a good degree of parallelism, as demonstrated by the values of the intercept $(b)$ that was not significantly different from 0 . Recovery was expressed as the regression curve between observed and expected hormone concentrations $\left(\left(H_{\mathrm{obs}}\right)=a\left(H_{\exp }\right)+b\right)$ measured in plasma samples spiked with known amount of DHEA-S. The angular coefficient (a) was not significantly different from 1 and the intercept $(b)$ was not significantly different from 0 indicating a good correspondence between expected and observed hormone concentrations. The sensitivity of the assay expressed as $90 \%$ of hormone bound was $3 \cdot 1 \mathrm{pg} /$ well. The intra- and inter-assay CV were $6 \cdot 7$ and $8 \cdot 3 \%$ respectively.

\section{Data analysis}

The pulsatile cortisol and DHEA release was analysed by the computer program 'Pulsar' based on the algorithm developed by Merriam \& Watcher (1982), which calculated the amplitude and frequency of hormone pulses, baseline concentrations and inter-peak intervals. Cut-off criteria $(G(1)$ to $G(5))$ were set to keep the probability of getting a false positive equal to or less than $5 \%$.

Data are expressed as mean \pm standard error. Hormone data underwent ANOVA by the general linear model procedure of SPSS. When appropriate, differences between means were analysed by Student $t$-test or Duncan test. In experiments 2, 3 and 5 , independent variables were the treatment $(T)$, sampling 
time (ST), animal (A) within $T$ and the $T \times \mathrm{ST}$ interaction. In experiment 3 , data measured before and after naloxone administration were recorded separately and were analysed by ANOVA. Terms of the models were $T$, the period $(P$, before naloxone administration versus after naloxone administration), A within $T$ and the $T \times P$ interaction. In experiment 4 , terms of the model were ST and A. Correlation between cortisol, DHEA and DHEA-S plasma concentrations were expressed as Pearson correlation coefficients (SPSS, Chicago, IL, USA).

\section{Results}

\section{Experiment 1: pattern of secretion}

The parameters describing the episodic release of cortisol, DHEA and DHEA-S over a period of $8 \mathrm{~h}$ (between 1000 and $1800 \mathrm{~h}$ ) in six non-pregnant dairy cows in their third month of lactation are reported in Table 1. The profiles of plasma DHEA and DHEA-S concentrations in the six cows are reported in Fig. 1. On average, plasma DHEA concentrations were significantly higher than plasma DHEA-S concentrations $(P<0 \cdot 001)$. The three steroids showed different patterns of episodic release. Plasma DHEA-S showed a weak but significant degree of correlation with both plasma cortisol $(R=-0 \cdot 264, P<0 \cdot 001)$ and DHEA $(R=0 \cdot 200, P<0 \cdot 001)$, while plasma cortisol and DHEA did not correlate each other.

\section{Experiment 2: response to single ACTH challenge}

The administration of the ACTH agonist induced a significant increase $(P<0 \cdot 01)$ of plasma cortisol within $1 \mathrm{~h}$ after injection. Circulating cortisol returned to basal values within $10 \mathrm{~h}$ after the drug administration (Fig. 2). On the contrary, both circulating DHEA and DHEA-S were not affected by the ACTH agonist (Fig. 2) and showed a significant cow effect $(P<0 \cdot 01)$. A weak but significant degree of correlation $(R=0 \cdot 202, P<0 \cdot 01)$ was found between DHEA and DHEA-S.

\section{Experiment 3: response to long-term ACTH administration}

During the ACTH phase, plasma cortisol was significantly higher $(P<0 \cdot 05 ; P<0 \cdot 01)$ from day 1 to day 7 , while plasma
DHEA was not affected by treatment (Fig. 3). No correlation was found between cortisol and DHEA.

Parameters describing cortisol release during frequent sampling (day 7) are reported in Table 2. Mean plasma cortisol was still significantly higher after the end of the ACTH phase $(P<0 \cdot 05)$. Cortisol peak amplitude, peak frequency and interpeak interval were affected by treatment $(P<0 \cdot 05)$. During the CTR phase, a cortisol pulse was observed within $30 \mathrm{~min}$ from naloxone administration in all animals. No apparent relationship between naloxone administration and cortisol pulse appearance was observed after the ACTH phase.

During frequent sampling, the mean concentrations and peak amplitude of plasma DHEA were significantly higher $(P<0 \cdot 05)$ in one cow, which was removed from the statistical analysis. The parameters describing DHEA release during frequent sampling (day 7) are reported in Table 3. Mean DHEA plasma levels were significantly higher after the ACTH phase $(P<0 \cdot 05)$, but no response to naloxone administration was observed after either CTR or ACTH phases.

During frequent sampling, plasma cortisol was not correlated to plasma DHEA in either the CTR or ACTH phases.

\section{Experiment 4: steroid variation around luteal regression}

Plasma concentrations of DHEA and DHEA-S were significantly different between individual cows $(P<0 \cdot 01)$. Circulating cortisol, DHEA and DHEA-S were not affected by the PG administration and no effect of the sampling time was observed (Fig. 4). A weak degree of correlation was found between DHEA and DHEA-S $(R=0 \cdot 216, P<0 \cdot 05)$.

\section{Experiment 5: response to continuous lactation}

During the prepartum period, plasma DHEA concentrations significantly $(P<0 \cdot 05)$ increased in both groups. In the CTRL group, plasma DHEA was significantly $(P<0 \cdot 05)$ higher between D-30 and D1 in comparison to D-60. In the MILK group, plasma DHEA was significantly $(P<0 \cdot 05)$ higher on D-7 than on D-60. Then, plasma DHEA decreased to concentrations lower than $1 \mathrm{nM}$ in both groups within 1 week after calving $(P<0 \cdot 01)$. Plasma DHEA concentrations were significantly lower in continuously milked cows at D-7 $(P<0 \cdot 05)$. As expected, plasma cortisol showed a significant

Table 1 Parameters describing the episodic release of cortisol, dehydroepiandrosterone (DHEA) and DHEA-sulphate (DHEA-S) over a period of $8 \mathrm{~h}$ (between 1000 and $1800 \mathrm{~h}$ ) in non-pregnant dairy cows $(n=6)$ in their third month of lactation

Mean concentration ( $\mathrm{nM})$

Peak amplitude (nM)

Peak duration (min)

Inter-peak interval $(\mathrm{min})$

Peak frequency (peak $n / 8 \mathrm{~h}$ )
Cortisol

DHEA

$$
\begin{gathered}
11 \cdot 8 \pm 1 \cdot 5 \\
18 \cdot 5 \pm 1 \cdot 6 \\
30 \pm 2 \\
104 \pm 9 \\
4 \cdot 3 \pm 0 \cdot 2
\end{gathered}
$$

$$
\begin{gathered}
2 \cdot 3 \pm 0 \cdot 1 \\
1 \cdot 2 \pm 0 \cdot 1 \\
24 \pm 2 \\
76 \pm 5 \\
6 \cdot 5 \pm 0 \cdot 3
\end{gathered}
$$

DHEA-S

$$
\begin{gathered}
0 \cdot 8 \pm 0 \cdot 2 \\
0 \cdot 4 \pm 0 \cdot 1 \\
22 \pm 2 \\
77 \pm 6 \\
5 \cdot 7 \pm 0 \cdot 4
\end{gathered}
$$



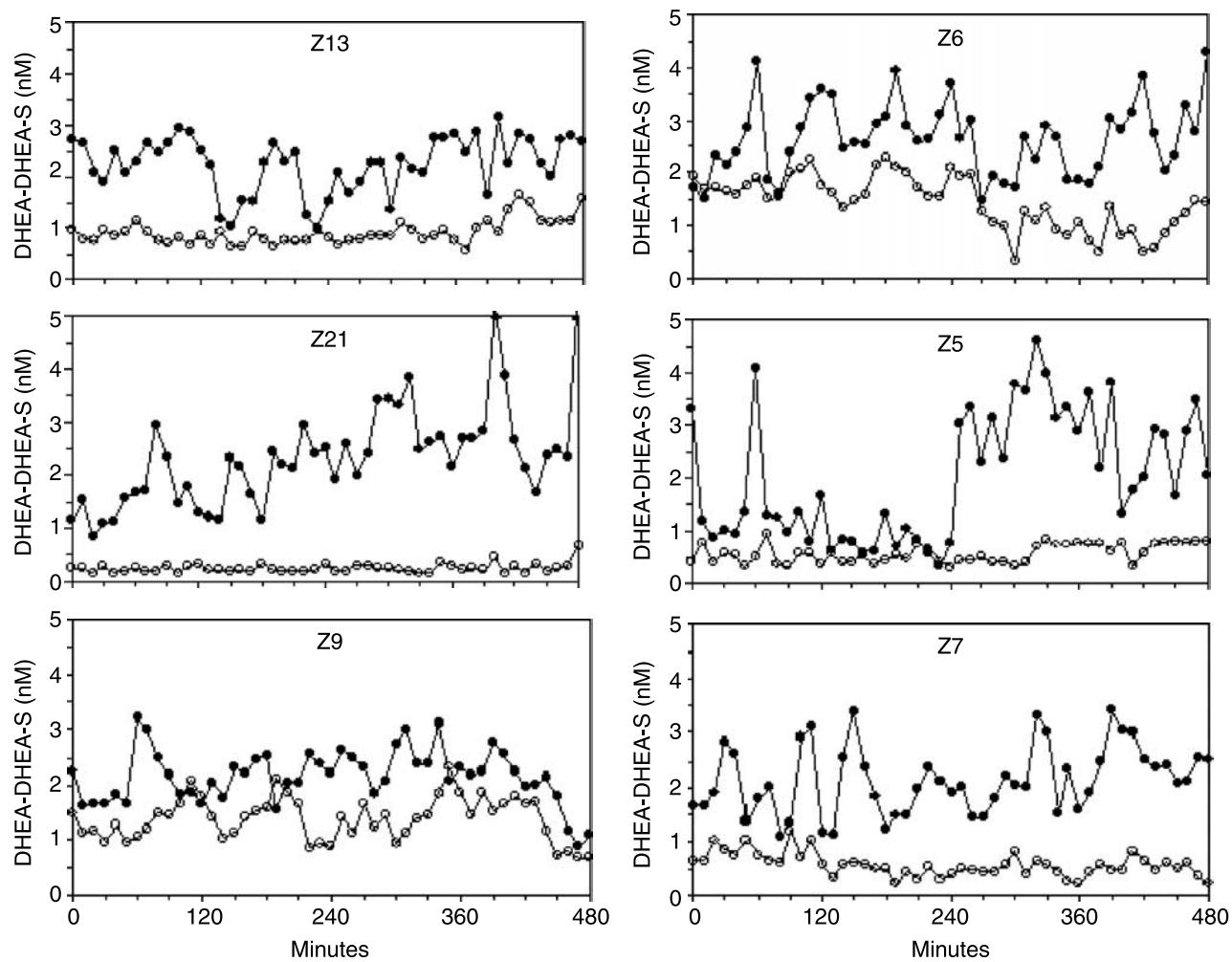

Figure 1 Plasma DHEA $(-)$ and DHEA-S $(O)$ concentrations in six non-pregnant dairy cows during the third month of lactation measured over a period of $8 \mathrm{~h}(1000-1800 \mathrm{~h})$ at 10-min intervals.

increase around parturition $(P<0 \cdot 01)$, but it was not affected by continuous milking (Fig. 5).

\section{Discussion}

Adrenal androgens play several important roles in primate mammals as they are implicated in the control of several biological functions (see Loria et al. 1996, Nestler 1996, Roberts 1999, Labrie et al. 2005). Also in laboratory animals, if administered at pharmacological doses, DHEA exerts its biological activities (Nestler 1996). Hence, this steroid can maintain a biological function also in non-primate mammals, despite the lower concentration observed in the circulation of those species in comparison with primates (Feher et al. 1977). However, little information about the physiological role of these steroids in non-primate mammals is available.

In the female bovine, circulating DHEA may serve as a source of precursor for androgen and oestrogen synthesis. For instance, the mammary gland can use DHEA to make androstene-3 $\beta, 17 \beta$-diol (AED; Belvedere et al. 1996), a metabolite with immunoenhancing activity (Loria et al. 1996). In addition, it was recently reported that cows with inflammatory foot lesions showed a $23 \%$ decrease in serum DHEA concentrations (Almeida et al. 2007), suggesting that DHEA is implicated in the inflammation process also in the bovine. Those observations, however, are not sufficient to hypothesise a biological role for DHEA in that species, and a better characterisation of the DHEA secretion is necessary.

In cows, circulating DHEA-S is significantly lower than DHEA, and DHEA release is very different among individual cows. Our data are consistent with previous observations in the bovine, where circulating DHEA-S was lower than in humans, and DHEA was more concentrated than DHEA-S in plasma (Feher et al. 1977). For this reason, in cows as in most non-primate mammals DHEA could be considered an indicator of the P450c17 enzyme activity and the most important circulating precursor of ectopic androgen and oestrogen synthesis, while the DHEA-S contribution as an androgen reservoir is rather limited.

The relationship between the secretion of DHEA and cortisol has not been fully understood. Our observations in cows showed that the secretion of cortisol, DHEA and DHEA-S is episodic. Cortisol and DHEA pulse frequency observed in cows was comparable with that observed in humans (Lejeune-Lenain et al. 1987), where DHEA and cortisol episodic secretions showed a strong overall synchronisation (Rosenfeld et al. 1975, Lejeune-Lenain et al. 1987). In particular, both steroids showed a bi-modal circadian pattern with a major acrophase in early morning and a late evening nadir, and a high degree of correlation and 

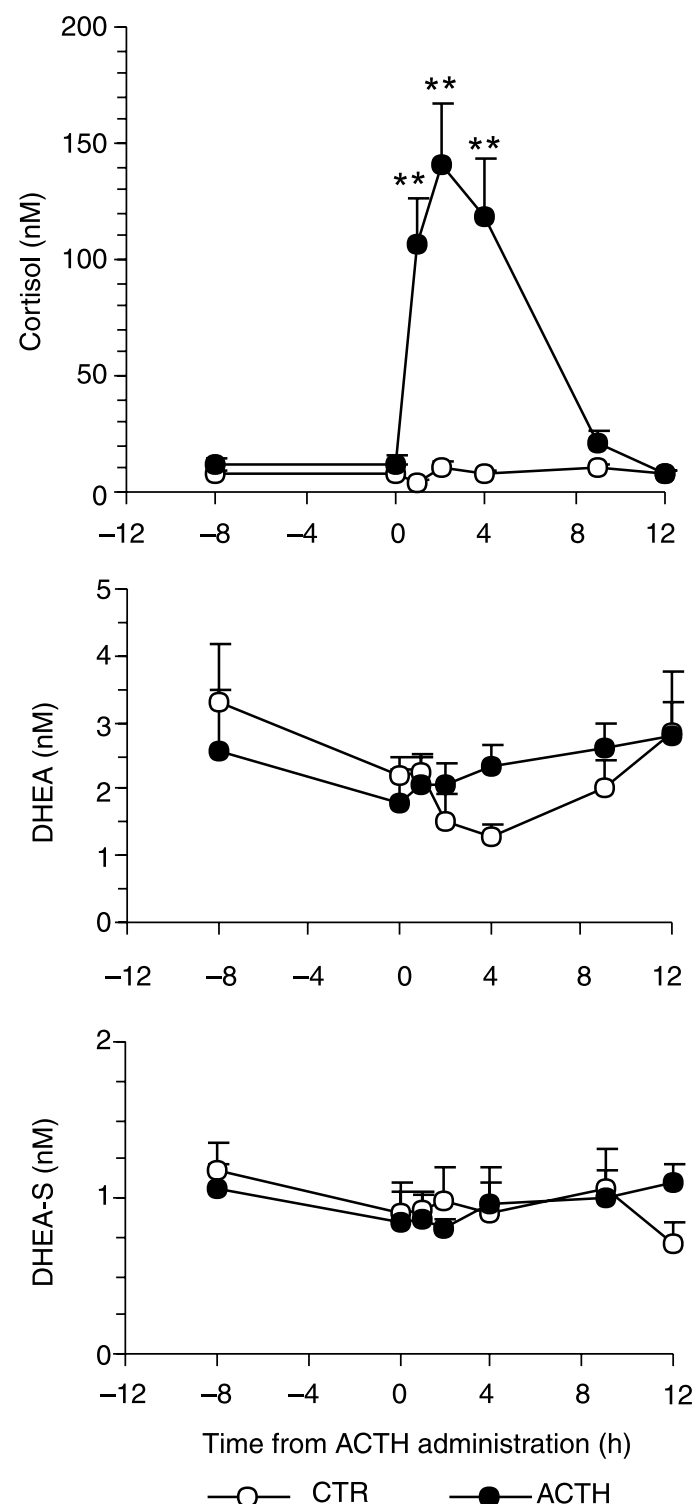

Figure 2 Cortisol, DHEA and DHEA-S plasma concentrations measured in four non-pregnant, non-lactating dairy cows treated with a single injection i.m. of saline $(C T R, O)$ or ACTH agonist $(\mathrm{ACTH}, \mathbf{O})$. The asterisks indicate statistically significant differences between CTR and ACTH (**P<0.01; Student $t$-test).

concomitance of episodic pulses (Lejeune-Lenain et al. 1987). In this study, frequent sampling was performed for $8 \mathrm{~h}$ from the morning to early afternoon, and this was not suitable to detect any circadian pattern of steroid release. During the observation period, however, no relationship between cortisol and DHEA secretion was found in the cow, suggesting that cortisol and DHEA release are desynchronised. We are not aware of any studies on the pattern of DHEA/DHEA-S release in non-primate mammals and, in this respect, our results bring an element of novelty.
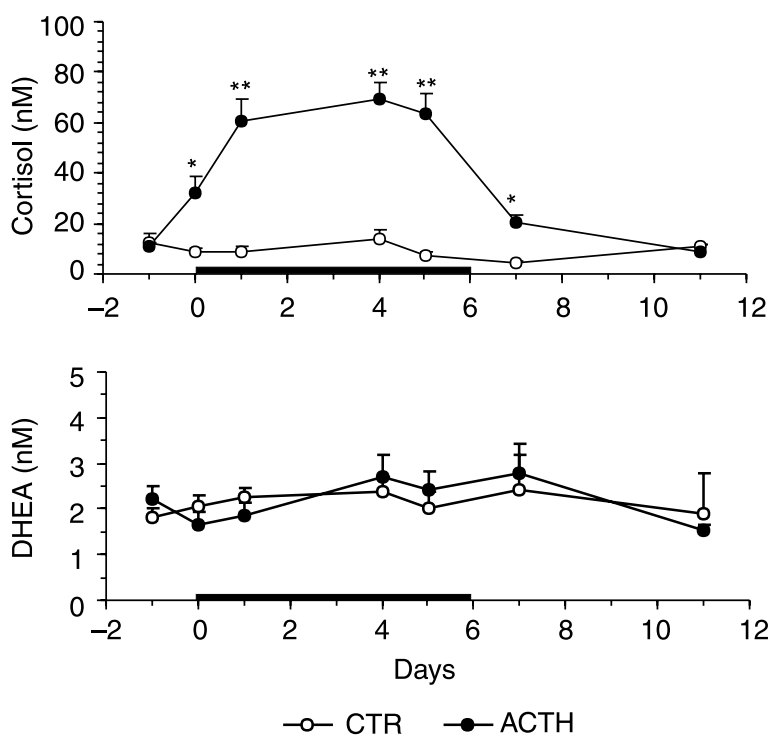

Figure 3 Plasma cortisol and DHEA concentrations measured in five non-pregnant, non-lactating cows treated with either saline $(\mathrm{CTR}, \mathrm{O})$ or an ACTH agonist $(\mathrm{ACTH}, \mathbf{O})$ administered every $12 \mathrm{~h}$ for 6 days. The black bar indicates the treatment administration. The asterisks indicate statistically significant differences between CTR and $\mathrm{ACTH}\left({ }^{*} P<0 \cdot 05 ;{ }^{* *} P<0 \cdot 01\right.$, Student $t$-test).

Although some in vitro studies showed that the bovine adrenals do secrete DHEA and respond to ACTH (Higuchi et al. 1985, Judd et al. 2000), our data strongly suggest that ACTH has a negligible effect on DHEA and DHEA-S release in the bovine in vivo.

In humans, DHEA secretion is responsive to ACTH stimulation (Parker 1999), although some observations are still controversial. In a dose-response study (Arvat et al. 2000), it was observed that plasma DHEA increased in response to a minimum dose of $0 \cdot 01 \mu \mathrm{g}$ ACTH and the response was similar in male and female subjects. Indeed, those authors concluded that DHEA was more sensitive than cortisol to ACTH stimulation.

However, in numerous situations described in humans, DHEA secretion is modulated separately from ACTH and diverges from that of cortisol (Odell \& Parker 1985, Azziz et al. 2001). For example, the adrenal of the adult human responds to stress with increased cortisol and diminished DHEA/DHEA-S secretion (Parker 1999). In another study performed in healthy reproductive-aged women (Azziz et al. 2001), it was observed that both basal and ACTH-stimulated DHEA release had a very high inter-subject variability. Those authors suggested that ACTH is only partially responsible for adrenal androgen secretion and factors other than ACTH are implicated in DHEA biosynthesis. A similar observation was reported in an early study (Odell \& Parker 1985) performed in castrated dogs treated with either synthetic ACTH or bovine pituitary extract. In that experiment, the bovine pituitary extract contained a substance that was more potent than ACTH in stimulating DHEA release in the castrated dogs (Odell \& 
Table 2 Parameters describing the episodic release of cortisol over a period of $8 \mathrm{~h}$ (between 1000 and $1800 \mathrm{~h}$ ) in non-pregnant dairy cows in their third month of lactation treated with either saline (CTR) or an adrenocorticotropic hormone (ACTH) agonist every $12 \mathrm{~h}$ for 6 days. After $4 \mathrm{~h}$, the cows received an injection of naloxone hydrochloride

CTR

\begin{tabular}{l} 
Before naloxone \\
\hline $7 \cdot 7 \pm 0 \cdot 6^{\mathrm{a}}$ \\
$11 \cdot 8 \pm 2 \cdot 6^{\mathrm{a}}$ \\
$29 \pm 2$ \\
$77 \pm 24^{\mathrm{b}}$ \\
$2 \cdot 8 \pm 0 \cdot 6^{\mathrm{a}}$
\end{tabular}

\section{ACTH}

\begin{tabular}{|c|c|}
\hline Before naloxone & After naloxone \\
\hline $59 \cdot 5 \pm 2 \cdot 4^{b}$ & $\overline{52 \cdot 5 \pm 1 \cdot 3^{b}}$ \\
\hline $33 \cdot 7 \pm 5 \cdot 1^{\mathrm{b}}$ & $25 \cdot 4 \pm 2 \cdot 4^{b}$ \\
\hline $24 \pm 3$ & $26 \pm 3$ \\
\hline $59 \pm 7^{a}$ & $51 \pm 6^{a}$ \\
\hline $4 \cdot 2 \pm 0 \cdot 2^{b}$ & $4 \cdot 2 \pm 0 \cdot 5^{b}$ \\
\hline
\end{tabular}

Mean concentration (nM)

Peak amplitude (nM)

Peak duration (min)

Inter-peak interval (min)

Peak frequency (peak $n / 4 \mathrm{~h})$

\begin{tabular}{l} 
After naloxone \\
\hline $8 \cdot 7 \pm 0 \cdot 8^{\mathrm{a}}$ \\
$13 \cdot 1 \pm 3 \cdot 5^{\mathrm{a}}$ \\
$27 \pm 8$ \\
$84 \pm 10^{\mathrm{b}}$ \\
$2 \cdot 2 \pm 0 \cdot 2^{\mathrm{a}}$
\end{tabular}

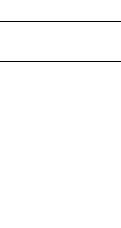

Different superscripts in the same row indicate significantly different means within treatment $\left({ }^{\mathrm{a}, \mathrm{b}} \mathrm{P}<0 \cdot 05\right.$; Duncan test).

Parker 1985). Nevertheless, subsequent reports failed to confirm the existence of such a substance (Nestler 1996).

The low ACTH effect on DHEA release in the cow was indirectly confirmed by naloxone administration. In the absence of adrenal stimulation by exogenous $\mathrm{ACTH}$, the injection of naloxone induced a rise of circulating cortisol within $30 \mathrm{~min}$, suggesting that the opioid antagonist dose was adequate to induce the release of $\mathrm{CRH} / \mathrm{ACTH}$. On the contrary, no DHEA response was observed following naloxone administration. Likely, naloxone acted centrally to overcome the opioid blockade of the CRH/ACTH release that induced a cortisol pulse, but it was not sufficient to stimulate DHEA release.

This is in agreement with what has been observed in other species. Human and animal studies have established that endogenous opioids can inhibit the hypothalamus-pituitaryadrenal (HPA) axis, and naloxone administration can stimulate ACTH and glucocorticoids secretion (Jessop 1999). On the other hand, acute opioid blockade failed to induce a DHEA-S response in humans, although it was associated with cortisol release (Ceballos et al. 2007).

In our work, when the cows were treated with exogenous ACTH, no cortisol or DHEA response to naloxone was detected. In this case, the ACTH treatment resulted in a massive cortisol release that, likely, masked the effect of naloxone. In addition, it is probable that exogenous ACTH impaired the feedback mechanism controlling $\mathrm{CRH} / \mathrm{ACTH}$ release, thus impeding any effect of naloxone. However, longterm exogenous ACTH administration resulted in an elevation of mean plasma concentration and pulse amplitude of DHEA, which were detectable only by frequent sampling.
Taken together, our data indicated that ACTH is not an important secretagogue for DHEA in the cow, and the adrenal contribution to plasma DHEA is scarce. However, the concentrations and secretory pattern of this steroid could be very different between individual animals, suggesting that some individuals may be more sensitive to ACTH. Alternatively, DHEA secretion could be more intense in particular physiological conditions not evidenced in this work, and it could be under the control of other mechanisms involving factors different from ACTH.

In humans, insulin can reduce circulating DHEA and DHEA-S (see Nestler 1996), but we are not aware about the relationship between insulin and DHEA in other species. In the periparturient cow, we found a small but significant negative correlation between insulin and DHEA $(R=-0 \cdot 225, P<0 \cdot 01$, unpublished data) that seems to agree with the observations made in humans. Nevertheless, endocrine modifications in the periparturient cow are so complex that it is unworthy to consider this observation physiologically relevant.

Other factors can affect adrenal steroidogenesis. For example, basal and ACTH-stimulated DHEA secretion from dispersed bovine adrenal cells can be enhanced by prolactin (Higuchi et al. 1985), and other proopiomelanocortin (POMC)-derived peptides, such as $\beta$-endorphin and joining peptide, can stimulate DHEA release from human adrenal cells (Clarke et al. 1996).

Also cytokines, such as interleukin-6 (IL-6) and tumour necrosis factor $\alpha(\mathrm{TNF} \alpha)$, act as intra-adrenal modulators of adrenal steroid secretions (Nestler 1996, Judd et al. 2000, Bornstein et al. 2004). IL-6 and TNF $\alpha$ are expressed in the

Table 3 Parameters describing the episodic release of dehydroepiandrosterone (DHEA) over a period of $8 \mathrm{~h}$ (between 1000 and $1800 \mathrm{~h}$ ) in non-pregnant dairy cows in their third month of lactation treated with either saline (CTR) or an adrenocorticotropic hormone (ACTH) agonist every $12 \mathrm{~h}$ for 6 days. After $4 \mathrm{~h}$, the cows received an injection of naloxone hydrochloride

\section{CTR}

Mean concentration $(\mathrm{nM})$

Peak amplitude (nM)

Peak duration (min)

Inter-peak interval (min)

Peak frequency (peak $n / 4 \mathrm{~h}$ )
Before naloxone

$$
1 \cdot 7 \pm 0 \cdot 1^{\mathrm{a}}
$$

$1 \cdot 2 \pm 0 \cdot 2^{\mathrm{a}}$

$22 \pm 3$

$73 \pm 13$

$3 \cdot 0 \pm 0 \cdot 4$
ACTH

\begin{tabular}{|c|c|}
\hline Before naloxone & After naloxone \\
\hline $2 \cdot 6 \pm 0 \cdot 1 b$ & $2 \cdot 4 \pm 0 \cdot 1 b$ \\
\hline $1 \cdot 5 \pm 0 \cdot 3^{b}$ & $1 \cdot 6 \pm 0 \cdot 1^{b}$ \\
\hline $22 \pm 3$ & $18 \pm 3$ \\
\hline $73 \pm 14$ & $71 \pm 7$ \\
\hline $2 \cdot 2 \pm 0 \cdot 4$ & $2 \cdot 5 \pm 0 \cdot 3$ \\
\hline
\end{tabular}

Different superscripts in the same row indicate significantly different means within treatment $\left({ }^{\mathrm{a}}{ }^{\mathrm{b}} \mathrm{P}<0 \cdot 05\right.$; Duncan test). 


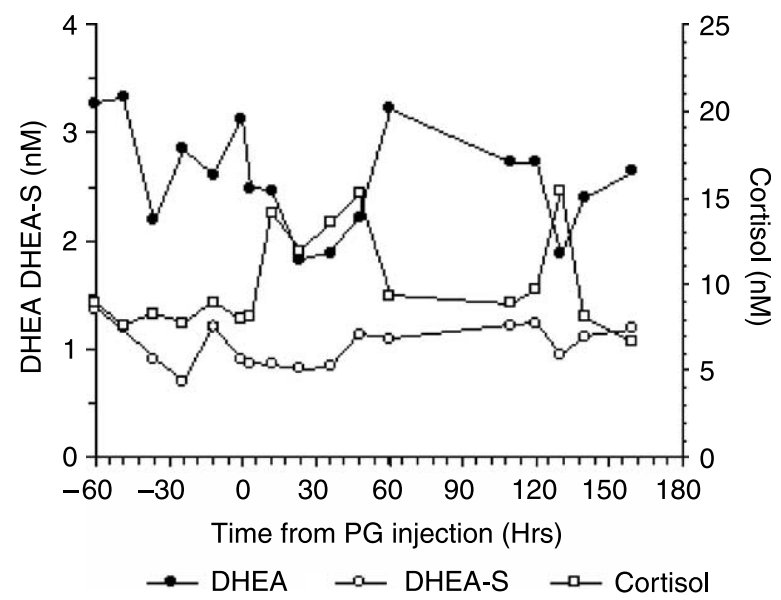

Figure 4 Cortisol $(\square)$, DHEA (O) and DHEA-S (O) plasma concentrations measured in four non-pregnant, non-lactating dairy cows around the time of induced luteal regression. Time 0 indicates the administration of the PGF $2 \alpha$ analogue. Pooled standard errors were cortisol $=0.64 \mathrm{nM}, \mathrm{DHEA}=0.11 \mathrm{nM}$ and DHEA-S $=0.05 \mathrm{nM}$.

adrenal cortex of human (Nestler 1996, Judd et al. 2000, Bornstein et al. 2004), rat and bovine (Judd et al. 2000), but they show opposite actions in the human and the bovine. IL-6 stimulates cortisol secretion from both human and bovine adrenal cells, and it has a stimulatory action on DHEA secretions in human adrenal cells. On the contrary, IL-6 exposure inhibits basal and ACTH-stimulated DHEA secretion from bovine adrenal cells, which is also inhibited by TNF $\alpha$ (Judd et al. 2000).
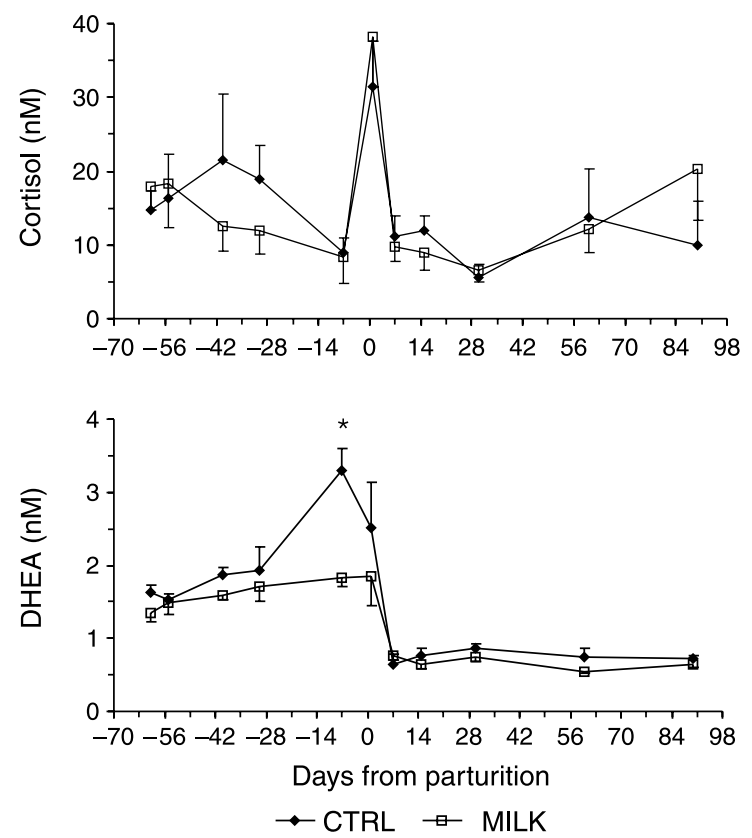

Figure 5 Plasma cortisol and DHEA concentrations measured in cows with a 55-day dry period (CTRL, $n=5,0)$ and continuously milked cows (MILK, $n=6, \square)$. The asterisk indicates a statistically significant difference between CTRL and MILK $\left({ }^{*} P<0 \cdot 05\right.$, Student $t$-test).
In the non-pregnant cow, DHEA and DHEA-S presumably originate from the adrenals (Higuchi et al. 1985, Judd et al. 2000) and the gonads (Wise et al. 1982), although the relative contribution of these tissues is difficult to extrapolate. However, our results suggest that the ovaries do not secrete an amount of DHEA capable of significantly affecting its plasma concentrations at the time of induced ovulation, possibly because at that time the steroid is used for oestrogen synthesis (Wise et al. 1982).

Likely, the most important source of DHEA in the cow is the placenta, which utilises mainly the $\Delta 5$ steroidogenic pathway to produce oestrogen (Geiser \& Conley 1998). The results of this and previous (Gabai et al. 2004) works indicate that the DHEA placental secretion increases in late pregnancy, probably depending upon the tissue mass (Geiser \& Conley 1998), and suddenly decreases after parturition, and the lactating mammary gland can affect the circulating levels of DHEA by converting it in other steroids (Belvedere et al. 1996). In other physiological conditions, plasma DHEA concentrations are rather stable, and they seem to be slightly altered only in the case of inflammatory events (Almaida et al. 2005).

For these reasons and because the dairy cow is often affected by inflammatory events during the last 2 months of pregnancy and the first 2 months of lactation (transition period) (Bionaz et al. 2007), in our opinion the investigation about the biological role of DHEA in this species should be focused particularly during the peripartum.

\section{Acknowledgements}

We are particularly grateful to $\mathrm{Mr}$ Tommaso Brogin for his skilled technical assistance.

\section{Funding}

The present work was supported by the Italian Ministry of University and Scientific Research (MUR, PRIN 2005). There is no conflict of interest that would prejudice the impartiality of the present research.

\section{References}

Almeida PE, Weber PSD, Burton JL \& Zanella AJ 2007 Depressed DHEA, and increased sickness response behaviours in lame dairy cows with inflammatory foot lesions. Domestic Animal Endocrinology (doi: 10.1016/j.doaniend.2006.11.006)

Aragno M, Mastrocola R, Medana C, Catalano MG, Vercellinatto I, Danni O \& Boccuzzi G 2006 Oxidative stress dependent impairment of cardiac-specific transcription factors in experimental diabetes. Endocrinology 147 5967-5974.

Arvat E, Di Vito L, Lanfranco F, Maccario M, Baffoni C, Rossetto R, Aimaretti G, Camanni F \& Ghigo E 2000 Stimulatory effect of adrenocorticotropin on cortisol, aldosterone, and dehydroepiandrosterone secretion in normal humans: dose-response study. Journal of Clinical Endocrinology and Metabolism 85 3141-3146. 
Azziz R, Fox LM, Zacur HA, Parker CR Jr \& Boots LR 2001 Adrenocortical secretion of dehydroepiandrosterone in healthy women: high variable response to adrenocorticotropin. Journal of Clinical Endocrinology and Metabolism 86 2513-2517.

Bastianetto S, Ramassamy C, Poirier J \& Quirion R 1999 Dehydroepiandrosterone (DHEA) protects hippocampal cells from oxidative stressinduced damage. Molecular Brain Research 66 35-41.

Baulieu EE 1998 Neurosteroids: a novel function of the brain. Psychoneuroendocrinology 23 963-987.

Belvedere P, Gabai G, Dalla Valle L, Accorsi PA, Trivoletti M, Colombo L \& Bono G 1996 Occurrence of steroidogenic enzymes in the bovine mammary gland at different functional stages. Journal of Steroid Biochemistry and Molecular Biology 59 339-347.

Bionaz M, Trevisi E, Calamari L, Librandi F, Ferrari A \& Bertoni G 2007 Plasma paraoxonase, health problems, inflammatory conditions and liver function in transition dairy cows. Journal of Dairy Science 90 1740-1750.

Bornstein SR, Rutkowski H \& Vrezas I 2004 Cytokines and steroidogenesis. Molecular and Cellular Endocrinology 215 135-141.

Ceballos NA, France CR \& al'Absi M 2007 Influence of naltrexone administration on dehydroepiandrosterone sulfate levels in male and female participants. Biological Psychology 74 414-416.

Clarke D, Fearon U, Cunnigham SK \& McKenna TJ 1996 The steroidogenic effects of $\beta$-endorphin and joining peptide: a potential role in the modulation of adrenal androgen production. Journal of Endocrinology 151 301-307.

Feher T, Bodrogi L, Feher KG, Poteczin E \& Kolcsey IS 1977 Free and solvolysable dehydroepiandrosterone and androsterone in blood of mammals under physiological conditions and following administration of dehydroepiandrosterone. Acta Endocrinologica 85 126-133.

Gabai G, Marinelli L, Simontacchi C \& Bono G 2004 The increase of plasma C195 steroids in subcutaneous abdominal and jugular veins of dairy cattle during pregnancy is unrelated to estrogenic activity. Steroids 69 121-127.

Gabai G, Mollo A, Marinelli L, Badan M \& Bono G 2006 Endocrine and ovarian responses to prolonged adrenal stimulation at the time of induced corpus luteum regression. Reproduction in Domestic Animals 41 485-493.

Geisert RD \& Conley AJ 1998 Secretion and metabolism of steroids in subprimate mammals during pregnancy. In The Endocrinology of Pregnancy, pp 291-318. Ed. FW Bazer. Totowa, NJ: Humana Press Inc.

Hansen PA, Han DH, Nolte LA, Chen M \& Holloszy JO 1997 DHEA protects against visceral obesity and muscle insulin resistance in rats fed a high-fat diet. American Journal of Physiology 273 R1704-R1708.

Higuchi K, Nawata H, Kato K \& Ibayashi H 1985 Ovine prolactin potentiates the action of adrenocorticotropic hormone on the secretion of dehydroepiandrosterone sulphate and dehydroepiandrosterone from cultured bovine adrenal cells. Hormone and Metabolic Research 17 451-453.

Hu Y, Cardounel A, Gursoy E, Anderson P \& Kalimi M 2000 Anti-stress effects of dehydroepiandrosterone. Biochemical Pharmacology 59 753-762.

Iwasaki Y, Asai M, Yoshida M, Nigawara T, Kambyashi M \& Nakashima N 2004 Dehydroepiandrosterone-sulphate inhibits nuclear factor- $\kappa \mathrm{B}$-dependent transcription in hepatocytes, possibly through antioxidant effect. Journal of Clinical Endocrinology and Metabolism 89 3449-3454.

Jessop DS 1999 Central non-glucocorticoid inhibitors of the hypothalamopituitary-adrenal axis. Journal of Endocrinology 160 169-180.

Judd AM, Call GB, Barney M, McIlmoil CJ, Balls AG, Adams A \& Oliveira GK 2000 Possible function of IL-6 and TNF as intraadrenal factors in the regulation of adrenal steroid secretion. Annals of New York Academy of Science $917628-637$.
Kochan Z \& Karbowska J 2004 Dehydroepiandrosterone up-regulates resistin gene expression in with adipose tissue. Molecular and Cellular Endocrinology 218 57-64.

Labrie F, Luu-The V, Belanger A, Lin S-X, Simard J, Pelletier G \& Labrie C 2005 Is dehydroepiandrosterone a hormone? Journal of Endocrinology 187 169-196.

Lejeune-Lenain C, Van Cauter E, Desir D, Beyloos M \& Franckson JRM 1987 Control of circadian and episodic variations of adrenal androgen secretion in man. Journal of Endocrinological Investigation 10 267-276.

Loria RM, Padgett DA \& Nuynh PN 1996 Regulation of the immune response by dehydroepiandrosterone and its metabolites. Journal of Endocrinology 150 S209-S220.

Maurice T, Urani A, Phan V-L \& Romieu P 2001 The interaction between neuroactive steroids and the $\sigma_{1}$ receptor function: behavioural consequences and therapeutic opportunities. Brain Research Reviews $\mathbf{3 7}$ 116-132.

Merriam GR \& Watcher KW 1982 Algorithms for the study of episodic hormone secretion. American Journal of Physiology 243 E310-E318.

Nestler JE 1996 Advances in understanding the regulation and biologic actions of dehydroepiandrosterone. Current Opinion in Endocrinology and Diabetes 3 202-211.

Odell WD \& Parker LN 1985 Control of adrenal androgen production. Endocrine Research 10 617-630.

Parker CR 1999 Dehydroepiandrosterone and dehydroepiandrosterone sulfate production in the human adrenal during development and aging. Steroids 64 640-647.

Pelissier M-A, Trap C, Malewiak M-I \& Morfin R 2004 Antioxidant effects of dehydroepiandrosterone and 7-hydroxy- dehydroepiandrosterone in the rat colon, intestine and liver. Steroids 69 137-144.

Roberts E 1999 The importance of being dehydroepiandrosterone sulfate (in the blood of primates). Biochemical Pharmacology 57 329-346.

Rosenfeld RS, Rosemberg BJ, Fukushima DK \& Hellman L 1975 24-Hour secretory pattern of dehydroisoandrosterone and dehydroisoandrosterone sulfate. Journal of Clinical Endocrinology and Metabolism 40 850-855.

SPSS 2005 14.0 Syntax Reference Guide., Chicago, IL: SPSS Inc..

Wise TH, Caton D, Thatcher WW, Rami Lehrer A \& Fields MJ 1982 Androstenedione, dehydroepiendrosterone and testosterone in ovarian vein plasma and androstenedione in peripheral arterial plasma during the bovine oestrous cycle. Journal of Reproduction and Fertility 66 513-518.

Zhao Z-Y, Xie Y, Fu Y-R, Li Y-Y, Bogdan A \& Touitou Y 2003 Circadian rhythm characteristics of serum cortisol and dehydroepiandrosterone sulfate in healthy Chinese men aged 30 to 60 years. A cross-sectional study. Steroids 68 133-138.

Zinder O \& Dar DE 1999 Neuroactive steroids: their mechanism of action and their function in stress response. Acta Physiologica Scandinavica 167 181-188.

Received in final form 15 June 2007

Accepted 18 June 2007

Made available online as an Accepted Preprint 19 June 2007 